\title{
The alignment of the ATLAS Inner Detector in Run 2
}

\author{
Giulia Ripellino* on behalf of the ATLAS Collaboration \\ KTH Royal Institute of Technology (SE) \\ E-mail: giulia.ripellinodcern.ch
}

\begin{abstract}
The ATLAS reconstruction of charged particle trajectories relies on the Inner Detector tracking system. The accuracy of the reconstruction is limited by the finite resolution of the detector elements and the imperfect knowledge about their positions. A precise alignment of the detector is therefore essential. Here, the strategy and the status of the Inner Detector alignment in ATLAS during the LHC Run 2 are presented and the alignment challenges related to the distortion of the new innermost Pixel layer, the IBL, are discussed.
\end{abstract}

Fourth Annual Large Hadron Collider Physics

13-18 June 2016

Lund, Sweden

${ }^{*}$ Speaker. 


\section{Introduction}

Charged particles are reconstructed using information from the Inner Detector system of the ATLAS experiment [1] at the LHC. The accuracy of the reconstruction is limited by the finite resolution of the detector elements and the imperfect knowledge about their positions. Misalignments of detector modules deteriorate the track resolution and can lead to systematic biases on the reconstructed track parameters. A precise alignment of the Inner Detector is therefore essential.

\section{The ATLAS Inner Detector}

The ATLAS Inner Detector (ID) is composed of three subsystems, the Pixel detector, the Semiconductor Tracker (SCT) and the Transition Radiation Tracker (TRT). The Pixel detector is composed of silicon pixel sensors, the SCT of silicon microstrip sensors and the TRT is a drift chamber composed of gas-filled straw tubes. All subsystems consist of a barrel with cylindrical layers and two end-caps made from disks of silicon sensors or TRT wheels. The whole ID is embedded in a $2 \mathrm{~T}$ axial magnetic field and is designed to reconstruct charged particles up to $|\eta|=2.5^{1}$. A new innermost layer in the Pixel detector, the IBL, was inserted for the LHC Run 2. This layer is composed of silicon pixel modules arranged on carbon fiber staves at a radius of $33 \mathrm{~mm}$ surrounding the beam pipe. The IBL provides an additional measurement point closer to the interaction point, thereby improving the impact parameter reconstruction and vertexing. Table 1 details the element size and resolution of each subsystem as well as the radii of the barrel layers.

\begin{tabular}{llll}
\hline Subdetector & Element size $[\mu \mathrm{m}]$ & Intrinsic resolution $[\mu \mathrm{m}]$ & Radius of the barrel layers $[\mathrm{mm}]$ \\
\hline IBL & $50 \times 250$ & $8 \times 40$ & 33.2 \\
Pixel & $50 \times 400$ & $10 \times 115$ & $50.5,88.5,122.5$ \\
SCT & 80 & 17 & $299,371,443,514$ \\
TRT & 4000 & 130 & $554-1082$ \\
\hline
\end{tabular}

Table 1: Summary of the main characteristics of the ID subdetectors. The intrinsic resolution of the IBL and the Pixel is reported along $r-\phi$ and $z$ and for SCT and TRT along $r-\phi$. For SCT and TRT the element sizes refer to the spacing of the readout strips and the diameter of the straw tubes, respectively.

\section{Alignment procedure}

The goal of the alignment procedure is to precisely determine the positions of all active detector elements. Each detector module is treated as a rigid body with six degrees of freedom (DoF), corresponding to the three possible translations and rotations. These DoF uniquely define the positions of the modules and are referred to as the alignment parameters. The alignment is performed

\footnotetext{
${ }^{1}$ ATLAS uses a right-handed coordinate system with its origin at the nominal interaction point (IP) in the centre of the detector and the $z$-axis along the beam pipe. The $x$-axis points from the IP to the centre of the LHC ring, and the $y$-axis points upward. Cylindrical coordinates $(r, \phi)$ are used in the transverse plane, $\phi$ being the azimuthal angle around the $z$-axis. The pseudorapidity is defined in terms of the polar angle $\theta$ as $\eta=-\ln \tan (\theta / 2)$.
} 
using a track-based technique which consists of finding the set of alignment parameters that minimizes the $\chi^{2}$,

$$
\chi^{2}=\sum_{\text {tracks }}[\mathbf{r}(\mathbf{a}, \boldsymbol{\tau})]^{\mathrm{T}} V^{-1}[\mathbf{r}(\mathbf{a}, \boldsymbol{\tau})]
$$

where $\mathbf{r}(\mathbf{a}, \boldsymbol{\tau})$ are the track-to-hit residuals, $\boldsymbol{\tau}$ are the track parameters, $\mathbf{a}$ are the alignment parameters and $V$ is the covariance matrix of the detector measurements. Using a large sample of good quality tracks, the $\chi^{2}$ is minimal for the solution to the alignment correction.

The alignment procedure is performed in three levels following the structure of the ID. Level 1 aligns all large structures; the IBL, the Pixel detector and the SCT and TRT barrels and end-caps. Level 2 corresponds to all silicon cylinders and disks and TRT modules and wheels. Finally, level 3 aligns the individual silicon modules and TRT straws. Table 2 details the number of alignable structures in each level for each subsystem.

\begin{tabular}{lrrrr}
\hline & \multicolumn{3}{c}{ Alignable Structures } \\
\hline & IBL & Pixel & SCT & TRT \\
\hline Level 1 & 1 & 1 & 3 & 3 \\
Level 2 & 1 & 9 & 22 & 96 \\
Level 3 & 280 & 1744 & 4088 & 350848 \\
\hline
\end{tabular}

Table 2: Number of alignable structures in each alignment level for each subsystem.

The basic quality of the alignment is assessed by means of track-hit residual distributions. A schematic drawing of the local residual vector and the transformation from the local coordinate system to the global coordinate system are shown in Figure 1.

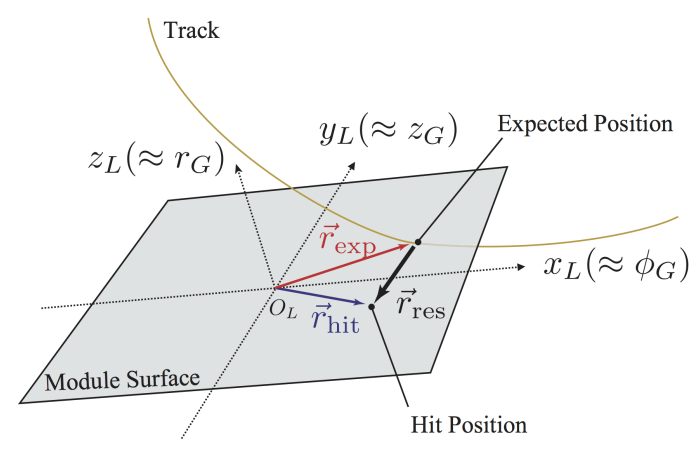

Figure 1: Coordinate system of the track-hit residual vector for silicon modules [2]. The local coordinate system is labeled $L$ and the ATLAS global coordinate system is labeled $G$.

\section{Alignment corrections to IBL distortions}

During the commissioning phase with cosmic data in 2015, a bowing of the IBL staves compared to the design geometry was observed. It is caused by a difference in the coefficients of 
thermal expansion of the IBL stave components and depends on the operating temperature [2]. In order to account for the bowing, new alignment parameters were introdued in the alignment procedure. Figure 2 shows the observed residual as a function of operating temperature after applying the alignment correction computed for data collected at $-20^{\circ} \mathrm{C}$.

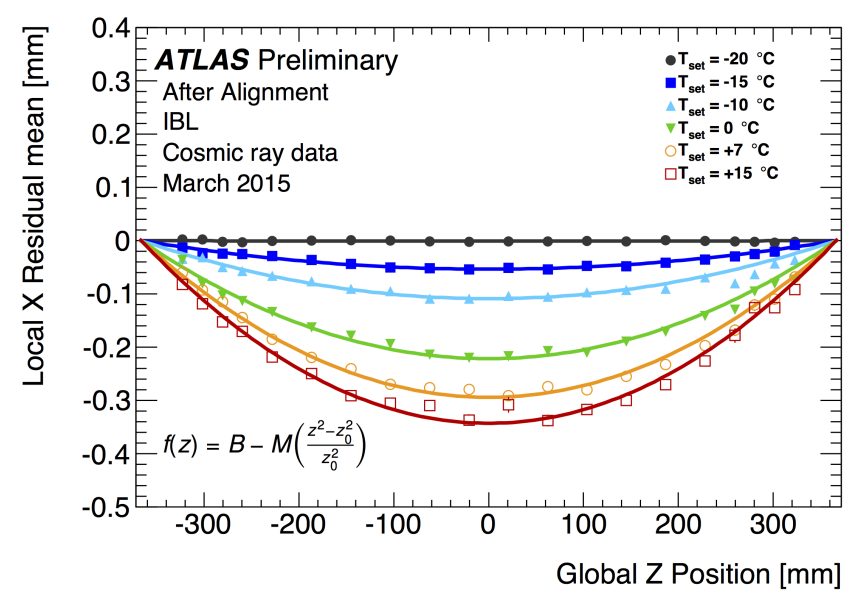

Figure 2: The track-to-hit residual mean in the local $x$-direction for the IBL [2]. The residual mean is averaged over all modules at the same global- $z$ positon. Alignment corrections derived at $-20{ }^{\circ} \mathrm{C}$ are applied to the local positions in the module frames.

In the collisions data taking the staves were further seen to bend due to temperature changes caused by an increased power consumption of the modules. The distortion varies with the integrated radiation dose and as a function of the LHC luminosity within a fill. A systematic timedependent alignment correction to mediate this effect was introduced in the ATLAS software and is now automated in the calibration of the data at the end of each run. With the new procedure there is no degradation of the physics performance. Figure 3 shows the time dependence of the distortion magnitude during a fill and the observed residual for default, fill-averaged and time-averaged alignment.
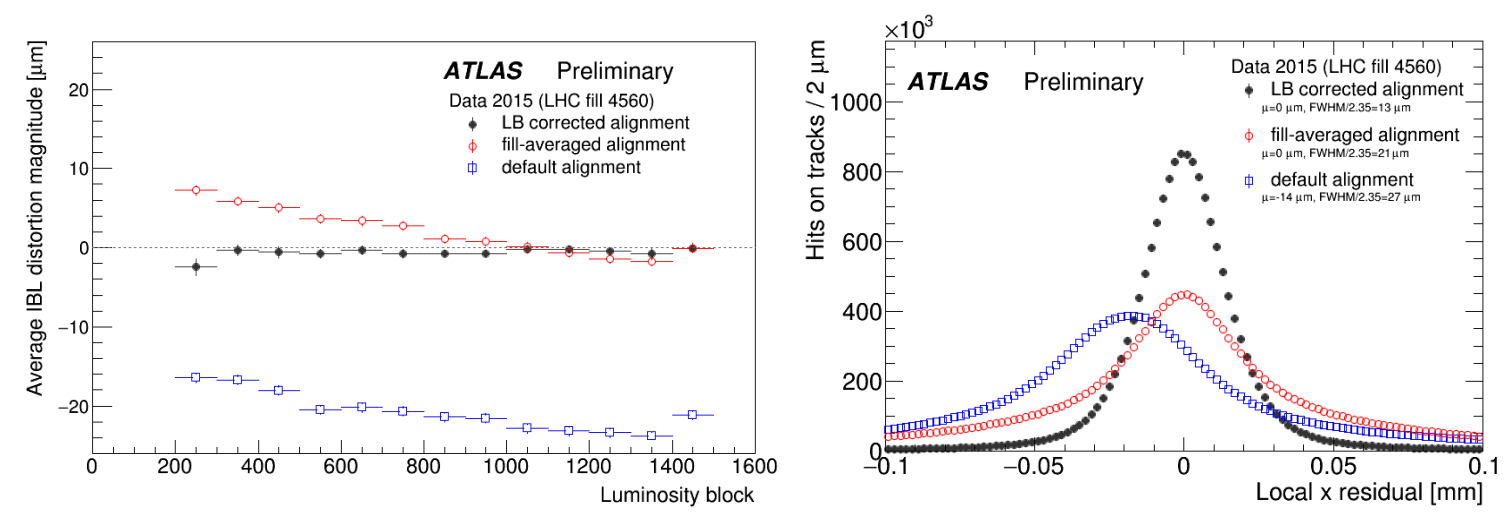

Figure 3: Distortion magnitude dependence in time (left) and track-hit residual mean in the local $x$-direction (right) for default, fill-averaged and time-averaged alignment [3]. 


\section{Alignment results in the 2015 and 2016 LHC runs}

The performance of the ID alignment in Run 2 is quantified by the residual distributions. Figure 4 compares the results between the 2015 and 2016 configurations for the IBL and the Pixel detector. The 2015 data is reprocessed with the final alignment configuration of 2015 . For the 2016 data, the commissioning is currently at an early stage but the alignment shows a similar quality.
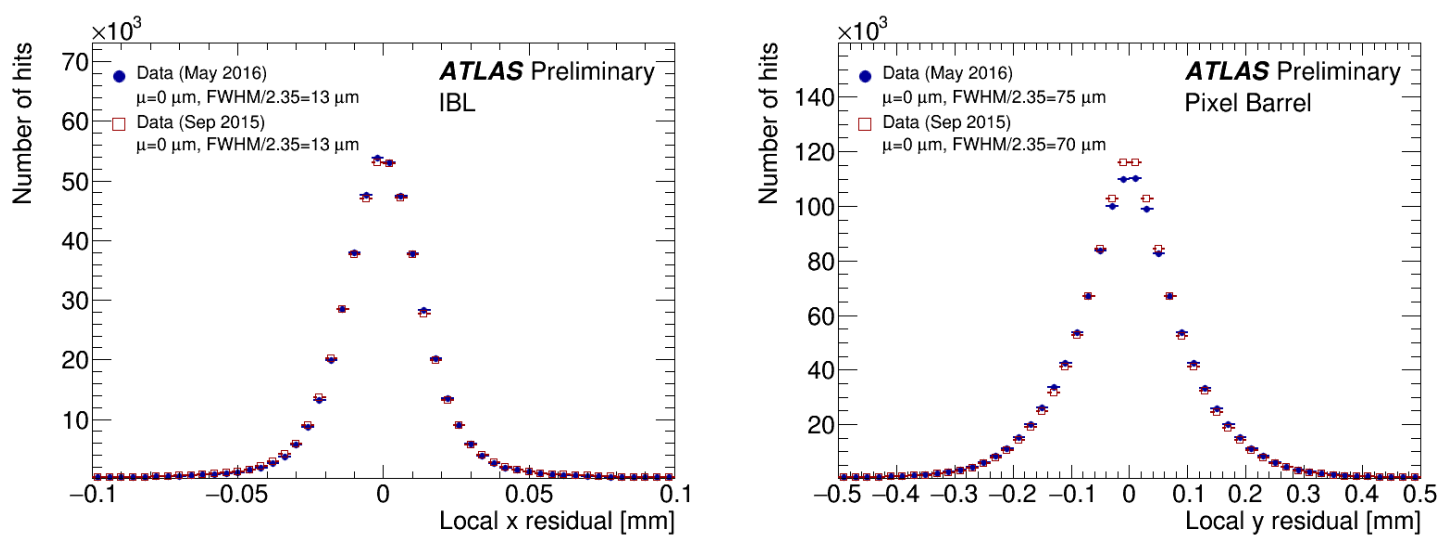

Figure 4: Local $x$ residual distributions for the IBL (left) and the Pixel detector barrel (right) for 2015 and 2016 data [4]. The parameter $\mu$ represents the mean of the distributions. The distributions are normalized to the same number of entries.

\section{Conclusions}

The ID alignment framework has succesfully been upgraded to cope with the Run 2 requirements. A new time-dependent scheme has been adopted to correct for the IBL misalignments and is now fully automatized in the calibration of the data at the end of each run. The commissioning of the 2016 data is currently at an early stage but the alignment shows a quality similar to the final 2015 configuration.

\section{References}

[1] ATLAS Collaboration, The ATLAS Experiment at the Large Hadron Collider, JINST 3 (2008) S08003

[2] ATLAS Collaboration, Study of the mechanical stability of the ATLAS Insertable B-Layer, ATL-INDET-PUB-2015-001, URL: https://cds.cern.ch/record/2022587

[3] ATLAS Collaboration, Time dependent alignment corrections to IBL distortions, IDTR-2015-01, URL: https://atlas.web.cern.ch/Atlas/GROUPS/PHYSICS/PLOTS/IDTR-2015-011

[4] ATLAS Collaboration, Alignment of the ATLAS Inner Detector with early 2016 LHC data, IDTR-2016-002, URL: https://atlas.web.cern.ch/Atlas/GROUPS/PHYSICS/PLOTS/IDTR-2016-002 